\title{
Sleep disorders in psychiatry
}

\author{
Hugh Selsick \& David 0’Regan
}

\begin{abstract}
SUMMARY
Sleep medicine is a truly multidisciplinary field that covers psychiatric, neurological and respiratory conditions. As the field has developed it has become increasingly clear that there is a great deal of overlap between sleep and psychiatric disorders and it is therefore essential for psychiatrists to have some knowledge of sleep medicine. Even those disorders, such as obstructive sleep apnoea, that may seem to be outside the remit of psychiatry can have complex and important interactions with psychiatric conditions. In this article we give a brief overview of the range of sleep disorders a psychiatrist might encounter, how they are recognised, investigated and treated, and how they relate to psychiatric conditions.
\end{abstract}

\section{LEARNING OBJECTIVES}

- Be aware of the range of sleep disorders that might be encountered in psychiatric practice

- Understand how these sleep disorders affect mental health

- Have a broad understanding of how these disorders are investigated and treated

\section{DECLARATION OF INTEREST}

H.S. has accepted speaker fees from Janssen Pharmaceuticals.

Sleep is a behaviour found throughout the animal kingdom and it occupies approximately a third of our lives. The preservation of this vulnerable state attests to its importance, yet we still do not fully understand its functions and mechanisms. Yet anyone who has experienced a sleep disorder will confirm how important good sleep is to health and quality of life. This is particularly true in mental healthcare, where poor sleep is a significant concern for many patients. It is likely that many people with sleep disorders will present to psychiatric services rather than sleep clinics and so it is essential that mental health workers are aware of these disorders and able to recognise them.

In this article we look at the six major categories of sleep disorder as defined in the International Classification of Sleep Disorders (ICSD-3; American Academy of Sleep Medicine 2014). The categories are:

- sleep-related breathing disorders

- central disorders of hypersomnolence
- parasomnias

- sleep-related movement disorders

- circadian rhythm sleep-wake disorders

- insomnia.

This article is not intended to be a detailed examination of these disorders, but rather a broad overview of the field designed to raise awareness of this vital and fascinating area.

\section{Sleep-related breathing disorders}

\section{What are they?}

Although there are several sleep-related breathing disorders the one most likely to be encountered in psychiatric practice is obstructive sleep apnoea (OSA). This is the repeated collapse of the pharyngeal portion of the airway during sleep, leading to partial or complete obstruction of the airway. This in turn leads to the restriction or cessation of airflow. Unlike central sleep apnoea, where respiration ceases because of a reduction in respiratory effort, the respiratory effort in OSA is maintained. The cessation of airflow can result in significant oxygen desaturation and is physiologically stressful. Ultimately, the apnoea will lead to an arousal when the patient will reopen their airway. As a result, sleep is disrupted by multiple stressful physiological events and multiple arousals. This usually (but not invariably) results in excessive daytime sleepiness and increased risk for numerous medical conditions, including hypertension, cardiac disease, diabetes and stroke (American Academy of Sleep Medicine 2014).

\section{Diagnosis and screening}

Often the first clue that a patient may have OSA is excessive daytime sleepiness. This can be measured quantitatively using the Epworth Sleepiness Scale, which asks patients about their likelihood of falling asleep in eight scenarios. Although there is some debate about the exact cut-off between normal and pathological sleepiness, a score of $10-11$ or above is considered indicative of excessive sleepiness. People with OSA are likely to be prolific snorers, although this is not always the case. They may report choking in their sleep or a bed partner may notice the apnoeas. OSA is more common in overweight individuals with thick necks or retrognathia and is more common with advancing age (Greenberg 2017).

Screening for OSA has become much cheaper and easier in recent years. Oximetry performed in the
Hugh Selsick is a consultant in sleep medicine and psychiatry and lead clinician at the Insomnia Clinic at the Royal London Hospital for Integrated Medicine/University College London Hospitals NHS Foundation Trust, and consultant in sleep medicine at the Sleep Disorders Centre at Guy's and St Thomas' NHS Foundation Trust, UK. He also runs the Sleep Working Group at the Royal College of Psychiatrists and is a council member of the Sleep Medicine Section at the Royal Society of Medicine, UK. David O'Regan is a consultant in psychiatry and sleep medicine at the Insomnia Clinic at the Royal London Hospital for Integrated Medicine/ University College London Hospitals NHS Foundation Trust, at the Sleep Disorder Centre at Guy's and St Thomas' NHS Foundation Trust and at Closed Loop Medicine, UK. He is a council member of the Sleep Medicine Section at the Royal Society of Medicine, UK. Correspondence Dr Hugh Selsick, Insomnia Clinic, Royal London Hospital for Integrated Medicine, 60 Great Ormond Street, London WC1N 3HR, UK. Email: hugh.selsick@nhs.net

Copyright and usage (C) The Royal College of Psychiatrists 2018 
home is now widely used as an initial screening tool and often this is sufficient to make the diagnosis. However, there are times when a more detailed respiratory study, which monitors respiratory effort and airflow in addition to oxygen saturation levels, or a full polysomnogram may be warranted. OSA is diagnosed if there are at least 15 obstructive respiratory events an hour. It is also diagnosed if there are more than five events an hour in the presence of daytime sleepiness, fatigue, observed or subjective apnoeas, hypertension, coronary artery disease, stroke, congestive heart failure, atrial fibrillation, type 2 diabetes, insomnia, cognitive dysfunction or a mood disorder (American Academy of Sleep Medicine 2014).

\section{Relationship to psychiatric disorders}

The fact that insomnia, cognitive dysfunction and mood disorders are among the diagnostic criteria for OSA gives an indication of the possible causative role of OSA in these disorders in some people. Cognitive impairment is a common finding in OSA. Some of this impairment is due to the direct effects of sleep disruption, but some of it is due to the hypoxic damage to the hippocampus and other structures. Surprisingly, at least some of this damage may be reversible with effective treatment of the OSA (Canessa 2011).

Cross-sectional studies have shown that depression is significantly more common in people with OSA. Although we do not know with certainty that OSA has a causative role in depression, given that $20-40 \%$ of people with OSA have depression (Harris 2009), it is clearly vital to screen OSA patients for depression. It is not yet possible to say whether treating OSA improves depression (Harris 2009).

Finally, given the propensity for many psychiatric drugs to cause weight gain, it is likely that many patients taking these medications will develop OSA as they put on weight. It is therefore important for psychiatrists to be vigilant for signs of OSA and to ask about these signs repeatedly over the years, particularly as increasing age is also risk factor for OSA.

\section{Treatment}

If weight is thought to be a factor, then weight loss may be curative. However, this is often very difficult, particularly for patients who are already tired and sleepy! In mild to moderate OSA a mandibular advancement splint, an intraoral device which advances the lower jaw, thus widening the airway, can be effective. However, for more severe cases the gold standard remains continuous positive airway pressure (CPAP) (Epstein 2009). CPAP involves sleeping with a mask that covers the nose or nose and mouth; a small air pump increases the air pressure within the mask so that the inspired air is at a higher pressure than the air in the room and this holds the airway open. Despite its efficacy, adherence is a problem with CPAP, often because of discomfort and claustrophobia. There is an increasing role for psychiatrists and psychologists in managing patient anxieties to improve adherence.

\section{Central disorders of hypersomnolence}

\section{What are they?}

Hypersomnolence is characterised by recurrent episodes of excessive daytime sleepiness or prolonged night-time sleep that is not restorative. The typical age at onset is between 17 and 24 years, it is present in $1 \%$ of the population and is equally common in males and females (Anderson 2007).

\section{Diagnosis and screening}

Individuals report a long nocturnal sleep (often more than $9 \mathrm{~h}$ a night) and feel unrefreshed on wakening. They frequently have difficulty in waking from sleep and may exhibit profound sleep inertia or 'sleep drunkenness', i.e. confusion, motor coordination difficulties and reduced alertness, which may linger for many hours. During the day, they are compelled to nap repeatedly, often at inappropriate times, such as at work or during a social event. The sleepiness is usually experienced as a gradual phenomenon, as opposed to a 'sleep attack' (narcolepsy). Daytime napping does not provide relief. Some individuals have a profound loss of functional ability across family, occupational and social settings (Moller 2008).

The excessive sleepiness is present despite a main sleep period lasting at least $7 \mathrm{~h}$. The symptoms must be present at least three times a week to receive a diagnosis of hypersomnolence disorder, which is further subclassified into acute (symptoms for less than 1 month), subacute (symptoms for between 1 and 3 months) and persistent (symptoms for more than 3 months) (American Psychiatric Association 2013).

Other contributing sleep disorders must be excluded, and to this end it is worth undertaking thorough sleep investigations, including actigraphy (for at least 2 weeks), followed by a nocturnal polysomnogram and the Multiple Sleep Latency Test.

In addition, other medical (e.g. obesity, multiple sclerosis, Parkinson's disease, sedating medications), psychiatric (e.g. affective disorders, Alzheimer's disease, sedating medications) and behavioural (e.g. alcohol, illicit substances) causes must be excluded; see Fig. 1 for a suggested approach.

Caution is advised against automatically ascribing excessive sleepiness only to sedative psychotropics, 
Excessive daytime somnolence

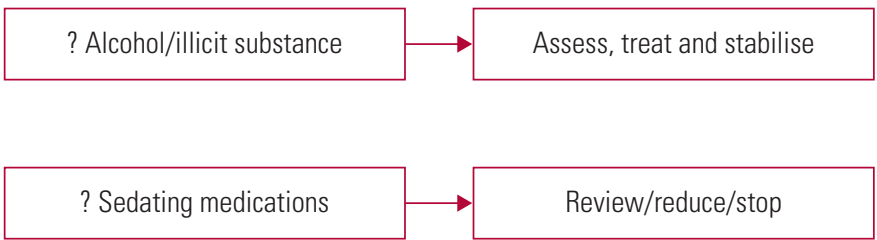

\begin{tabular}{|c|c|}
\hline ? Cognitive disorder & Assess \\
\cline { 3 - 3 }
\end{tabular}
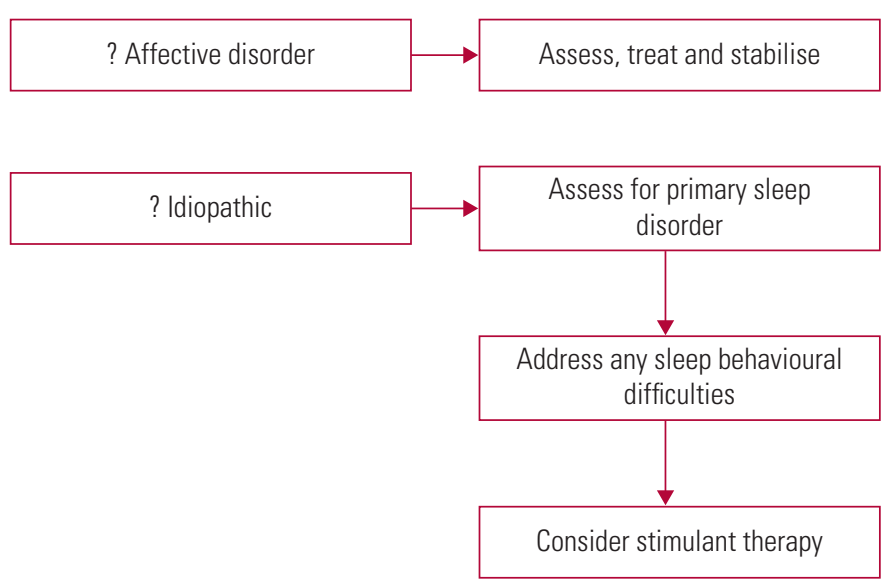

Approach to hypersomnolence.

and it is worth excluding other possible contributors (e.g. a comorbid sleep disorder). More often in clinical practice, the underlying causes of excessive daytime somnolence tend to be multifactorial.

\section{Relationship to psychiatric disorders}

Individuals with hypersomnolence disorder may exhibit other psychiatric symptoms, including anxiety, irritability, anergia, bradyphrenia, slowed speech, loss of appetite and cognitive difficulties (Bassetti 1997).

As noted above, hypersomnolence may be associated with affective disorders, substance-related disorders and dementias and can be a side-effect of psychotropic medications.

\section{Treatment}

Stimulants (e.g. modafinil, methylphenidate and dexamfetamine) are the mainstay of treatment (Ali 2009). Alerting antidepressants may also be used. Behavioural strategies such as avoiding activities that delay bedtime may provide some relief. The
Epworth Sleepiness Scale can be used to monitor response to treatment.

\section{Parasomnias}

What are they?

Parasomnias are undesirable physical, experiential or behavioural phenomena that occur at sleep onset and during sleep - things that go bump in the night. They are mainly classified into nonrapid eye movement (non-REM), rapid eye movement (REM) and other parasomnias. Box 1 lists some common parasomnias.

\section{Diagnosis and screening}

Non-REM parasomnias typically occur within the first third of the night. They tend to commence in childhood and diminish with age. There may be up to three episodes per night, and their frequency may vary. Stress, alcohol and sleep deprivation tend to trigger increases in frequency in adults. Typically, a person will have their eyes open during an episode, may leave the bed and will be 
BOX 1 Common parasomnias

Non-REM

- Confusional arousals

- Sleepwalking

- Sleep terrors

REM

- REM-sleep behavioural disorder

- Nightmare disorder

- Isolated sleep paralysis

Other

- Sleep enuresis

- Sleep-related groaning

- Sleep disassociation

amnesic for the event the next morning. There is often a positive family history.

REM parasomnias occur in the second half of the night. There may be one or two episodes per night, the person will typically have their eyes closed and they rarely leave the bed.

Diagnosis is usually clinical, but may be challenging, as there is often poor patient recall, no collateral/witness account and routine investigations may be normal.

Table 1 offers guidance on distinguishing between sleep (night) terrors and nightmares. In distinguishing nocturnal panic, there is often a history of daytime anxiety, panic or agoraphobia, and in the night there is physiological warning (e.g. racing heart, shortness of breath, dry mouth), accompanied by an intense feeling of anxiety.

REM-sleep behavioural disorder (RBD) is more common in men over 50 years of age. In the disorder,

\section{TABLE 1 Main differences between sleep (night) terrors (a non-REM parasomnia) and nightmares (a REM parasomnia)}

\begin{tabular}{|lll|}
\hline & \multicolumn{2}{c|}{ Parasomnia } \\
\cline { 2 - 3 } Characteristic & Sleep terror & Nightmare \\
\hline Time of night & First third & Last third \\
Movements & Common & Rare \\
Severity & Severe & Mild \\
Vocalisation & Common & Rare \\
Autonomic discharge & Severe and intense & Mild \\
Amnesia & Present & Absent \\
State on waking & Disoriented/confused & Oriented \\
Leave the bed & Common & Very rare \\
\hline
\end{tabular}

REM, rapid eye movement.
REM atonia is lost, so the individual may act out their dreams. The person usually has little, if any, knowledge of their actions, but may recall dream mentation, which often involves fighting others, for example to protect their home or loved ones from intruders.

Video polysomnography may be useful, particularly in excluding any other sleep disorder that may be triggering the parasomnia. For example, OSA may underlie a presentation of RBD (i.e. pseudo-RBD), so in a patient with risk factors for sleep-disordered breathing, home pulse oximetry may be a useful screen. Patients withdrawing from alcohol and benzodiazepines may also present with a pseudo-RBD. Parasomnia behaviour may be difficult to differentiate from nocturnal frontal lobe epilepsy, and again video polysomnography may be helpful. In patients presenting with adult-onset nonREM parasomnias, a magnetic resonance imaging (MRI) scan of the brain is useful in excluding structural precipitants.

\section{Relationship to psychiatric disorders}

Given their often bizarre motor and/or emotional behaviours, parasomnias may be easily confused with psychiatric disorders. For some, there may be associations with psychiatric disorders and their treatments. In adults with sleep terrors, for example, $30 \%$ will have a comorbid psychiatric disorder and 50\% will have experienced a stressful life event in the preceding year (Ohayon 1999). Antidepressants can unmask a subclinical RBD or make it worse (bupropion is perhaps the exception) (Postuma 2013).

$\mathrm{RBD}$ is the most robust non-motor predictor of developing Parkinson's disease and it predicts early cognitive impairment and visual hallucinations. Around 50\% of patients with RBD have mild cognitive impairment at diagnosis; and by 13 years postdiagnosis, 65\% will have developed Parkinson's disease and/or dementia (Fulda 2011).

\section{Treatment}

Most non-REM parasomnias are clinically mild and self-limiting. Patient education, reassurance, safety advice and avoidance of triggers will often suffice. Any contributing comorbid sleep disorder should be addressed. Cognitive-behavioural therapy for insomnia (CBT-I, discussed below) may be useful in stabilising sleep-wake patterns and in teaching relaxation/anxiety-reduction techniques. Patients at risk of injury or harm may require low-dose clonazepam at night (e.g. Markov 2006). The Paris Arousal Disorders Severity Scale may be useful in monitoring response to treatment. 
In $\mathrm{RBD}$, patient education and safety measures are important. In violent $\mathrm{RBD}$, it is worth advising that bed partners sleep apart (if possible) until the condition is controlled. Any contributing medications (e.g. antidepressants) should be reduced, stopped or switched. Patients with additional excessive daytime somnolence should be investigated further (see 'Diagnosis and screening' above). If treatment is necessary, there is randomised control evidence for clonazepam, but it causes daytime sedation in $66 \%$ of patients, will worsen OSA and symptoms return on stopping it (Anderson 2009). Melatonin (e.g. modified release tablets $2-8 \mathrm{mg}$ ) is a safer option; it works by restoring REM atonia (McGrane 2015). Finally, cholinesterase inhibitors may be useful in patients with Parkinson's disease and RBD (Di Giacopo 2012).

In nightmare disorder, offending medications (e.g. beta-blockers, L-dopa) should be reviewed. Imagery rehearsal therapy and CBT may be helpful. Medically, trazodone and prazosin may be considered (Aurora 2010).

\section{Sleep-related movement disorders: restless legs syndrome and periodic limb movements in sleep}

\section{What are they?}

Restless legs syndrome (RLS) is a common neurological disorder, which is clinically diagnosed on history (see below). Periodic limb movements in sleep (PLMS) is a related sleep movement disorder, defined by criteria set out by the American Academy of Sleep Medicine (2014), and requires a nocturnal polysomnogram for diagnosis. Up to $80 \%$ of people with RLS exhibit PLMS (Montplaisir 1998), though the reciprocal relationship is not as robust.

\section{Diagnosis and screening}

Patients with sleep movement disorders may present with insomnia, as limb movements prevent sleep onset or disturb sleep maintenance. People with PLMS (and their bed partners) are often unaware of these repetitive leg movements, which disturb sleep maintenance and stage 3 sleep (the most refreshing part of sleep). As a result, they frequently present with excessive daytime somnolence, although to their knowledge they have no apparent difficulties with sleep maintenance.

The essential criteria for a diagnosis of RLS can be summarised as unpleasant sensations in the legs (e.g. pain, tingling, feelings of electricity or whirring), which are associated with the urge to move, which cannot be ignored, and which are partially or wholly relieved by movement (Allen 2014). The symptoms show diurnal variation, and most commonly come on in the evening when the person is at rest. They may also be episodic in nature. Although the legs are most commonly affected, symptoms may arise from any muscle group, for example restless arms or back. To make the diagnosis, other medical and behavioural factors must be excluded, such as renal failure, diabetes and low ferritin levels.

Although PLMS is diagnosed by polysomnography, it can vary significantly from night to night. Therefore, a negative polysomnogram in the context of a convincing history should be investigated further, for example via domiciliary lower limb actigraphy.

\section{Relationship to psychiatric disorders}

Sleep movement disorders and psychiatric disorders are frequently comorbid (Haba-Rubio 2005). Moreover, many psychotropics can unmask or exacerbate these conditions.

Predictably, all typical antipsychotics with dopamine receptor blocking properties will exacerbate sleep movement disorders. Within the atypical class, case reports of olanzapine and risperidone worsening RLS have been published (e.g. Basu 2014). There are insufficient data available about the effects of other antipsychotics, but as a partial dopamine agonist, theoretically aripiprazole may have a favourable effect on RLS.

Various tricyclic antidepressants, selective serotonin reuptake inhibitors (SSRIs) and serotoninnoradrenaline reuptake inhibitors (SNRIs) exacerbate RLS or PLMS; mirtazapine in particular has been strongly implicated (Rottach 2008). In contrast, bupropion, a dopamine agonist, may help alleviate RLS symptoms.

Centrally acting emetics such as promethazine and over-the-counter antihistamines such as diphenhydramine have also been implicated.

Given the effects of these medications on RLS symptoms, it is important to screen for RLS before starting psychotropic therapy. Up to $66 \%$ of patients with moderate to severe RLS will also experience depression and panic disorder (Becker 2006). In a patient with severe RLS and mild depressive symptoms, it may be reasonable to treat the RLS first to see whether improvements in sleep and daytime functioning lead to a resolution of depressive symptoms.

In general, anticonvulsants that are associated with pain relief ameliorate RLS symptoms. Gabapentin and carbamazepine are second-line agents in the treatment of RLS, and valproic acid might also be helpful in reducing symptoms.

\section{Treatment}

First, all secondary causes must be excluded. In particular, for patients with a ferritin level less 
than $50 \mu \mathrm{g} / \mathrm{L}$, iron supplementation should be commenced. If possible, any associated psychotropic should be stopped or switched to one that is not known to exacerbate sleep movement disorders.

Consideration could then be given to a small evening dose of a dopamine agonist (e.g. ropinirole). Compulsive gambling, overeating and hypersexuality have been associated with dopamine agonist treatment of Parkinson's disease and, to a lesser degree, RLS (Moore 2014). Therefore, in patients with impulse-control disorder or affective disorders (e.g. bipolar affective disorder) clinicians should be aware of the potential for initiating or exacerbating impulsive behaviour or mood symptoms. Similarly, dopamine agonist treatment may unmask an underlying psychotic illness. Dopamine agonists should never be given to patients with an active psychosis, and caution should be exercised in those with a history of psychosis.

Treatment with alpha-2-delta agonists such as pregabalin may be especially useful, as these will also help protect stage 3 sleep and reduce night-time anxiety, which can be a problem for many patients.

The International RLS Study Group's Restless Legs Syndrome Rating Scale may be useful is monitoring response to treatment.

\section{Circadian rhythm sleep-wake disorders}

\section{What are they?}

Our internal biological master clock is located in the suprachiasmatic nucleus $(\mathrm{SCN})$ in the brain. The SCN has direct neural connections with other brain areas and also communicates with the rest of the body by controlling the release of the endocrine hormone melatonin from the pineal gland. The SCN is active in the day and in the presence of light. When the SCN is active it inhibits melatonin secretion. In the evening the $\mathrm{SCN}$ becomes less active and the pineal gland starts to secrete melatonin. Melatonin further inhibits the SCN and therefore promotes its own secretion in a positive feedback loop. Melatonin receptors are widely expressed throughout our body and melatonin acts as a signal that 'tells our body it is night'. In the morning the SCN becomes active again and melatonin levels drop. Circadian rhythm disorders (CRDs) occur when this internal biological clock is disrupted or when it becomes misaligned with the outside world. Table 2 describes the various disorders that fall into this category.

\section{Diagnosis and screening}

Generally, patients present with a stable or recurrent pattern of sleep-wake disturbance that leads to sleepiness when the person wants to be awake and alertness when the person wants to sleep. This is often mistaken for insomnia. However, patients with insomnia often report being 'tired but wired' when awake, whereas those with a CRD are more likely to be sleepy.

The diagnosis is usually made on the basis of history. Clinicians should ask about typical times of sleep onset and waking when there are no external time constraints and whether these times change when there are external constraints, such as having to rise at a particular time for work. They should determine what time of day the patient feels most alert and most sleepy. A sleep diary for at least 7 days (but ideally longer) that captures work/ school and non-work/non-school days is immensely helpful. A visual diary in which the patient colours in the times they are asleep and awake can be more useful than a written diary, as it makes it easier to discern patterns in the sleep (Fig. 2).

Actigraphy, which involves wearing an accelerometer on the wrist, can be used to obtain objective, longitudinal data on the patient's sleep cycle (Littner 2003). Actigraphy generates a visual representation of the sleep pattern similar to the diary in Fig. 2 and can be particularly useful if the patient is unable to keep a diary.

\section{Relationship to psychiatric disorders}

The circadian rhythm controls not only physiological variables and alertness but also mood. Our mood is lowest around the time of the circadian nadir (when alertness is lowest) in the early hours of the morning (Wirz-Justice 2008). Most of us sleep through this mood nadir and are therefore rarely exposed to it. However, in delayed sleep-wake phase disorder (DSWPD) the delayed circadian rhythm means that the mood nadir occurs later in the day, when patients are often awake. It is therefore postulated that the high rates of depression in DSWPD (Kripke 2008) may be due to the repeated exposure to this mood nadir. Treating the DSWPD may not only treat the sleep disorder but also reduce depressive symptoms (Borodkin 2010). In patients with non-24-hour sleep-wake disorder there is also a high rate of depression and in some the severity of that depression will vary with the sleep phase, being greater when sleep occurs in the day and lesser when sleep occurs at night (Borodkin 2010).

It is often assumed that the sleep-wake cycle disruption commonly seen in schizophrenia is due to lack of structure, social isolation or other lifestyle factors. However, circadian rhythm disorders are present in a significant proportion of people with schizophrenia and it may be this underlying biological disorder that leads to the sleep disruption and lifestyle changes (Wulff 2010).

DSWPD is also common in children and adults with attention-deficit hyperactivity disorder $(\mathrm{ADHD})$ 


\begin{tabular}{|c|c|c|}
\hline Disorder & Description & Comments \\
\hline $\begin{array}{l}\text { Delayed sleep-wake phase } \\
\text { disorder }\end{array}$ & $\begin{array}{l}\text { The body clock is delayed relative to the outside world. The person is } \\
\text { unable to initiate sleep until well past the conventional bedtime } \\
\text { and sleeps into the late morning or the afternoon if allowed to }\end{array}$ & $\begin{array}{l}\text { More common in adolescents. The person is most alert at night and } \\
\text { struggles to function in the morning. In addition to the circadian } \\
\text { misalignment these individuals are often sleep deprived as they } \\
\text { fall asleep late, but are forcibly woken early to attend school, } \\
\text { work, etc. }\end{array}$ \\
\hline $\begin{array}{l}\text { Advanced sleep-wake } \\
\text { phase disorder }\end{array}$ & $\begin{array}{l}\text { The body clock is advanced relative to the outside world. The person } \\
\text { struggles to stay awake until the conventional bedtime and wakes } \\
\text { very early in the morning }\end{array}$ & $\begin{array}{l}\text { More common in older adults. Results in less educational or } \\
\text { occupational dysfunction (they are rarely late for work) and so may } \\
\text { be less likely to come to medical attention }\end{array}$ \\
\hline $\begin{array}{l}\text { Irregular sleep-wake } \\
\text { rhythm disorder }\end{array}$ & $\begin{array}{l}\text { Irregular sleep-wake episodes throughout the } 24 \mathrm{~h} \text {. The person has } \\
\text { broken sleep at night and excessive sleepiness with multiple naps } \\
\text { in the day. There is a loss of any clear circadian rhythm }\end{array}$ & $\begin{array}{l}\text { More common in neurodegenerative disorders, e.g. dementia and } \\
\text { developmental disorders }\end{array}$ \\
\hline $\begin{array}{l}\text { Non-24-hour sleep-wake } \\
\text { rhythm disorder }\end{array}$ & $\begin{array}{l}\text { The circadian rhythm, which is slightly longer (or, more rarely, shorter) } \\
\text { than } 24 \mathrm{~h} \text {, is not synchronised with the outside world. As a result, } \\
\text { the sleep period gets progressively later (or progressively earlier) } \\
\text { and rotates around the clock }\end{array}$ & $\begin{array}{l}\text { More common in blind people as they are unable to use light to } \\
\text { synchronise the body clock. As their sleep time drifts around the } \\
\text { clock the person will have periods where they are sleeping at a } \\
\text { normal time and periods when they are sleeping at an } \\
\text { inappropriate time }\end{array}$ \\
\hline Shift work disorder & $\begin{array}{l}\text { Working shifts that sometimes overlap with the normal sleep time } \\
\text { leads to insomnia when trying to sleep between shifts, excessive } \\
\text { sleepiness when awake or both. Total sleep time is reduced }\end{array}$ & $\begin{array}{l}\text { Most commonly reported in workers who do night shifts, early morning } \\
\text { shifts or rotating shifts. Can compromise safety in the workplace } \\
\text { and while commuting to and from work }\end{array}$ \\
\hline Jet lag disorder & $\begin{array}{l}\text { Insomnia or excessive sleepiness with reduced total sleep time } \\
\text { following travel across at least two time zones }\end{array}$ & $\begin{array}{l}\text { It is easier to adjust to westward travel than eastward travel as it is } \\
\text { easier to delay the body clock than to advance it }\end{array}$ \\
\hline $\begin{array}{l}\text { Circadian sleep-wake } \\
\text { phase disorder not } \\
\text { otherwise specified }\end{array}$ & $\begin{array}{l}\text { Sleep-wake disruption due to circadian misalignment or alteration of } \\
\text { the circadian rhythm that does not meet criteria for any of the } \\
\text { above disorders }\end{array}$ & Can occur in neurodegenerative disorders \\
\hline
\end{tabular}

After American Academy of Sleep Medicine (2014).

and treatment of the DSWPD can lead to improvements in the ADHD symptoms and daytime functioning (Borodkin 2010).

\section{Treatment}

The treatment of circadian rhythm disorders is complex but relatively free of significant side-effects. The mechanism of the circadian rhythm indicates that there are two ways of intervening in the patient's circadian rhythm. The first is to give the patient melatonin and the second is to use light (preferably sunlight or a seasonal affective disorder lamp). Melatonin will pull the sleep period towards it, i.e. if it is taken before the habitual sleep time the patient will fall asleep earlier; if it is taken after the habitual waking time it will delay their subsequent sleep period. Light has the opposite effect: light before sleep will delay sleep, whereas light on waking will advance the sleep (Morgenthaler 2007). In practice the treatment is more subtle than this and the timing of the melatonin or light is absolutely critical, particularly as the specific timings may change from day to day. For example, in our clinic we advise patients with DSWPD to take their melatonin $6 \mathrm{~h}$ earlier than the time that they fell asleep the night before. As the therapy takes effect they will start to fall asleep earlier and therefore they will take their melatonin progressively earlier as well.

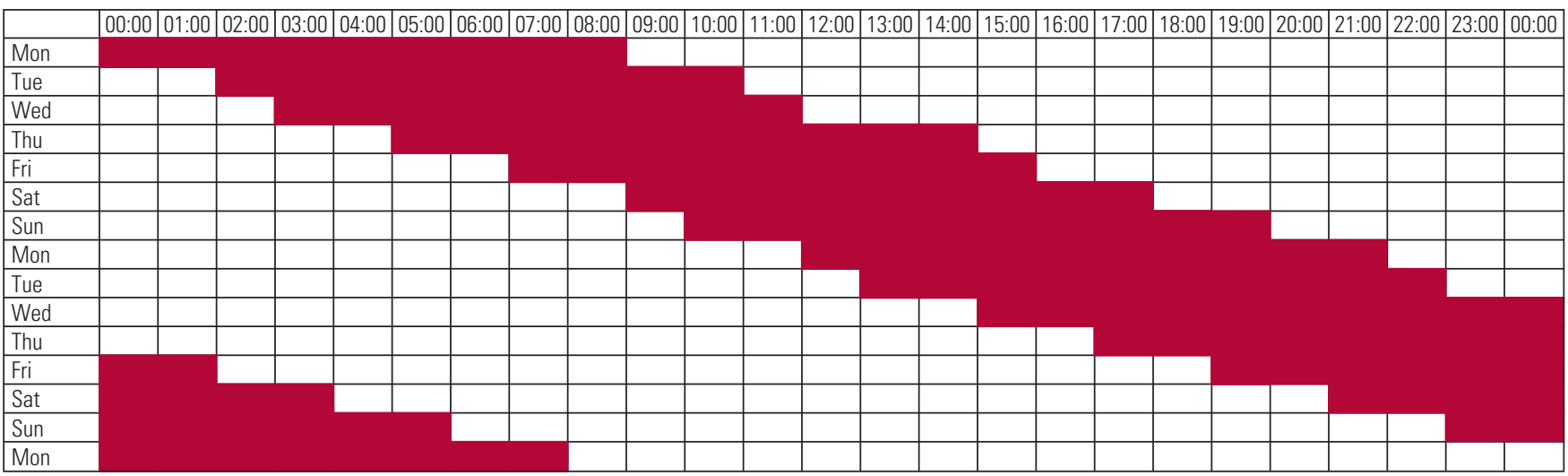

An example of a sleep diary in which the patient coloured in the hours she was asleep in red and left the hours she was awake blank. This makes it easy to see the progressive delay in her sleep time, indicating a non-24-hour sleep-wake rhythm disorder. 


\section{Insomnia}

\section{What is it?}

Insomnia is difficulty getting to sleep or staying asleep (either because of waking during the night or early morning waking) that leads to dissatisfaction or concern about sleep or to daytime consequences such as mood disturbances, irritability, fatigue, sleepiness, impaired social and occupational functioning, or impairments in memory, concentration or attention (American Academy of Sleep Medicine 2014). The critical point is that there must be some adverse consequence to the sleep pattern. Someone who habitually sleeps for a short period or has fragmented sleep without daytime consequences is likely to be someone who simply needs less than the average amount of sleep (a short sleeper) and would not be considered to have a sleep disorder. It is also important to point out that insomnia is only diagnosed if the sleep complaint is not entirely due to inadequate sleep opportunity (insufficient sleep syndrome) or adverse environmental conditions such as excessive noise and light or an unsafe environment.

For chronic insomnia to be diagnosed the sleep disturbance and daytime symptoms must occur at least three times a week for at least 3 months (American Academy of Sleep Medicine 2014).

\section{Diagnosis and screening}

Insomnia is generally obvious to both patients and clinicians. Where clinicians often err is in either failing to exclude the differential diagnoses or in assuming that the insomnia is a symptom of another condition rather than a disorder in its own right.

Insomnia is a clinical diagnosis and a good history is usually all that is required to make the diagnosis. Objective sleep studies are rarely needed in insomnia assessments unless the clinician suspects that there are other sleep disorders present, such as PLMS or OSA. A sleep diary for at least 7 days can be very helpful, particularly as patients often tend to describe the worst-case scenario rather than their typical symptoms when asked to describe their sleep. The clinician should enquire about work patterns, environmental factors, daytime naps, caffeine and alcohol consumption, medication use and what activities aside from sleep occur in the bedroom.

Differential diagnoses that must be discounted include CRDs, RLS and PLMS, OSA and insufficient sleep (behaviourally induced). If these diagnoses are missed, then treating the disorder as an insomnia is likely to lead to suboptimal results or may even make the disorder worse. For example, antihistamines or sedative antidepressants are often prescribed for insomnia, but they can exacerbate RLS. For the same reason, if the patient does not respond to standard insomnia treatments the clinician should consider the possibility that they might have a different sleep disorder that was missed at the initial assessment.

A common mistake is to assume that the insomnia is simply a symptom of depression and that treating the depression will resolve the insomnia as well. As a result, many insomnia patients are treated with antidepressants such as SSRIs, which may actually make the insomnia worse. It is also enormously irritating for euthymic patients with insomnia to be told that they are depressed; they feel very misunderstood and frustrated that their primary complaint is not being directly addressed. Where insomnia and depression coexist, the insomnia should not be thought of as being secondary to the depression: rather, they should be seen as comorbid conditions. This approach is reflected in DSM-5, which has deliberately omitted the terms 'primary' and 'secondary' from the insomnia diagnoses (American Psychiatric Association 2013).

\section{Relationship to psychiatric disorders}

Of all the sleep disorders, insomnia is the one that can most clearly lay claim to being a psychiatric disorder in its own right. It is often driven by psychological factors and can have a similar impact on quality of life as major depressive disorder (Katz 2002). There is also a high rate of insomnia in psychiatric patients: for example, one study found that $60 \%$ of new referrals to a psychiatric clinic complained of insomnia (Okuji 2002) and another reported that $40 \%$ of patients with insomnia had a comorbid psychiatric disorder (Roth 2007). All psychiatric patients should therefore be screened for insomnia.

There is mounting evidence that insomnia is a risk factor for the subsequent development of other psychiatric disorders, particularly depression. Where insomnia and depression coexist, the insomnia occurs first in 69\% of patients (Johnson 2006) and insomnia may predict subsequent depression decades after the insomnia develops (Chang 1997), making it unlikely that it is just a prodromal symptom of depression. Patients with insomnia have a two-fold risk of developing depression (Baglioni 2011) and so they should be monitored closely. However, if the insomnia resolves, the risk of subsequent depression is reduced (Franzen 2008). Similarly, the presence of insomnia predicts a poorer response to psychological and pharmacological treatments for depression, and if insomnia remains as a residual symptom when depression remits it predicts a higher rate of relapse. And although there is some debate about whether insomnia is an independent risk factor for suicide, it is undoubtedly a marker for 
increased suicide risk (Franzen 2008). Fortunately, there is also evidence that treating the insomnia can lead to improved outcomes in the comorbid psychiatric condition (Manber 2009) and we discuss this in the next section.

Finally, it is important to remember that many psychiatric drugs have insomnia as a side-effect and clinicians should be cognisant of this when prescribing. As a rule of thumb any drug that increases serotonergic, noradrenergic, dopaminergic or cholinergic tone has the potential to promote wakefulness and cause insomnia. However, in some drugs with antihistaminergic, anti-adrenergic or anticholinergic properties the sedative effects may counterbalance or outweigh the stimulant effects.

\section{Treatment}

There are two approaches to the treatment of insomnia: medication and cognitive-behavioural therapy for insomnia (CBT-I). The majority of hypnotics licensed for use in insomnia (benzodiazepines, zopiclone and zolpidem) act by enhancing the inhibitory effects of gamma-aminobutyric acid (GABA) via the benzodiazepine receptor. A slow-release version of melatonin and some antihistamines are also licensed for insomnia treatment. These medications can be safe and effective if used in the right way, at the right dose and with the right patient, but studies of long-term efficacy (more than a year) are lacking.

Probably the most important factor to consider when prescribing is the half-life of the drug. Most hypnotics have a quick onset of action but there are large differences in their half-lives and duration of action. Shorter-acting drugs (e.g. melatonin and zolpidem) are good for sleep-onset insomnia, but may be less effective in sleep-maintenance insomnia. Longer-acting drugs (e.g. zopiclone and temazepam) are likely to be good for sleep onset and maintenance, but carry the risk of morning hangover and should be used with caution in people who drive.

In patients with insomnia and comorbid psychiatric conditions there is good evidence that hypnotics are effective at treating the insomnia. Hypnotics may also accelerate or improve the response to standard treatments for generalised anxiety disorder, depression or schizophrenia (Pollack 2008; Fava 2011; Tek 2014).

Hypnotics are only licensed for short-term use in the UK. The problem with this is that the drugs only work for as long as they are being taken (Riemann 2009) and insomnia is often a chronic condition. We therefore find ourselves in a situation where there are only short-term licensed medications for a long-term problem. Fortunately, there is an alternative approach to the long-term treatment of insomnia in the form of CBT-I.
CBT-I is an effective, evidence-based treatment for insomnia that can be delivered one-to-one, in groups or online. Indeed, in chronic insomnia CBT-I should be the first-line treatment. It has also been shown to be an effective intervention in patients with comorbid psychiatric conditions (Taylor 2014) and there is mounting evidence that CBT-I can have a treatment effect on those comorbid psychiatric disorders. For example, a study comparing online CBT for depression with online CBT for insomnia in people with comorbid depression and insomnia found that the CBT for insomnia was more effective than the CBT for depression at improving sleep and that the two interventions were equally effective at treating the depression (Blom 2015). Similarly, CBT-I has been shown to reduce sleep complaints and persecutory delusions in people with psychosis (Myers 2011).

\section{Conclusions}

There is a wide variety of sleep disorders and they all have the potential to affect mental health. Sleep can also be disrupted by psychiatric disorders and the treatments for those disorders. It may seem counter-intuitive that what happens in a person's airways or legs could have an impact on their mental health, but sleep medicine demonstrates how closely integrated our physiological and psychological systems can be. This is a rapidly growing area and we anticipate that sleep medicine will play an increasing role in managing psychiatric conditions in the future.

Sleep is important to patients and having a psychiatrist take a genuine interest in their sleep makes them feel understood and cared for. By the time patients have their sleep disorder properly investigated and treated they will often have endured many years of suffering and frustration. Psychiatrists may be the first, or perhaps the only, professionals to ask a patient about their sleep and so it essential that they are able to recognise sleep disorders. By identifying and treating their patients' sleep disorders, psychiatrists have the opportunity to dramatically improve their patients' quality of life, their physical well-being and their mental health.

\section{References}

Ali M, Auger RR, Slocumb NL, et al (2009) Idiopathic hypersomnia: clinical features and response to treatment. Journal of Clinical Sleep Medicine, 5 : 562-8.

Allen RP, Picchietti DL, Garcia-Borreguero D, et al (2014) Restless legs syndrome/Willis-Ekbom disease diagnostic criteria: updated International Restless Legs Syndrome Study Group (IRLSSG) consensus criteria - history, rationale, description, and significance. Sleep Medicine, 15: 860-73.

American Academy of Sleep Medicine (2014) International Classification of Sleep Disorders (3rd edn). American Academy of Sleep Medicine.

American Psychiatric Association (2013) Diagnostic and Statistical Manual of Mental Disorders (5th edn) (DSM-V). American Psychiatric Publishing.
MCO answers

1 c 2 b $3 d \quad 4$ e 5 b 
Anderson KN, Pilsworth S, Sharples LD, et al (2007) Idiopathic hypersomnia: a study of 77 cases. Sleep, 30: 1274-81.

Anderson KN, Shneerson JM (2009) Drug treatment of REM sleep behavior disorder: the use of drug therapies other than clonazepam. Journal of Clinical Sleep Medicine, 5: 235-9.

Aurora RN, Zak RS, Auerbach SH, et al (2010) Best practice guide for the treatment of nightmare disorder in adults. Journal of Clinical Sleep Medicine, 6: 389-401.

Baglioni C, Battagliese G, Feige B, et al (2011) Insomnia as a predictor of depression: a meta-analytic evaluation of longitudinal epidemiological studies. Journal of Affective Disorders, 135: 10-9.

Bassetti C, Aldrich MS (1997) Idiopathic hypersomnia. A series of 42 patients. Brain, 120: 1423-35.

Basu A, Kundu S, Khurana H (2014). Olanzapine-induced restless leg syndrome: a case report and review of literature. Indian Journal of Pharmacology, 46: 450-2.

Becker PM (2006) The biopsychosocial effects of restless legs syndrome (RLS). Neuropsychiatric Disease and Treatment, 2: 505-12.

Blom K, Jernelöv S, Kraepelien M, et al (2015) Internet treatment addressing either insomnia or depression, for patients with both diagnoses: a randomized trial. Sleep, 38: 267-77.

Borodkin K, Dagan Y (2010) Circadian rhythm disorders. In Foundations of Psychiatric Sleep Medicine (eds J Winkelman, D Plante): 186-201. Cambridge University Press.

Canessa N, Castronovo V, Cappa SF, et al (2011) Obstructive sleep apnea: brain structural changes and neurocognitive function before and after treatment. American Journal of Respiratory and Critical Care Medicine, 183: 1419-26.

Chang PP, Ford DE, Mead LA, et al (1997) Insomnia in young men and subsequent depression: the Johns Hopkins Precursors Study. American Journal of Epidemiology, 146: 105-14.

Di Giacopo R, Fasano A, Ouaranta D, et al (2012) Rivastigmine as alternative treatment for refractory REM behavior disorder in Parkinson's disease. Movement Disorders, 27: 559-61.

Epstein LJ, Kristo D, Strollo PJ Jr, et al (2009) Clinical guideline for the evaluation, management and long-term care of obstructive sleep apnea in adults. Journal of Clinical Sleep Medicine, 5: 263-76.

Fava M, Schaefer K, Huang H, et al (2011) A post hoc analysis of the effect of nightly administration of eszopiclone and a selective serotonin reuptake inhibitor in patients with insomnia and anxious depression. Journal of Clinical Psychiatry, 72: 473-9.

Franzen PL, Buysse DJ (2008) Sleep disturbances and depression: risk relationships for subsequent depression and therapeutic implications. Dialogues in Clinical Neuroscience, 10: 473-81.

Fulda S (2011) Idiopathic REM sleep behaviour disorder as a long-term predictor of neurodegenerative disorders. EPMA Journal, 2: 451-8.

Greenberg H, Lakticova V, Scharf SM (2017) Obstructive sleep apnea: clinical features, evaluation, and principles of management. In Principles and Practice of Sleep Medicine (6th edn) (eds MH Kryger, T Roth, WC Dement): 1110-24. Elsevier.

Haba-Rubio J (2005) Psychiatric aspects of organic sleep disorders. Dialogues in Clinical Neuroscience, 7: 335-46.

Harris M, Glozier N, Ratnavadivel R, et al (2009) Obstructive sleep apnea and depression. Sleep Medicine Reviews, 13: 437-44.

Johnson EO, Roth T, Breslau N (2006) The association of insomnia with anxiety disorders and depression: exploration of the direction of risk. Journal of Psychiatric Research, 40: 700-8.

Katz D, McHorney C (2002) The relationship between insomnia and health-related quality of life in patients with chronic illness. Journal of Family Practice, 51: 229-35.

Kripke DF, Rex KM, Ancoli-lsrael S, et al (2008) Delayed sleep phase cases and controls. Journal of Circadian Rhythms, 6: 1-14.
Littner M, Kushida CA, Anderson WM, et al (2003) Practice parameters for the role of actigraphy in the study of sleep and circadian rhythms: an update for 2002. Sleep, 26: 337-41.

Manber R, Chambers AS (2009) Insomnia and depression: a multifaceted interplay. Current Psychiatry Reports, 11: 437-42.

Markov D, Jaffe F, Doghramji K (2006) Update on parasomnias: a review for psychiatric practice. Psychiatry, 3: 69-76.

McGrane IR, Leung JG, St Louis EK, et al (2015) Melatonin therapy for REM sleep behavior disorder: a critical review of evidence. Sleep Medicine, 16: 19-26.

Moller HJ, Lam S (2008) Quality of life in excessive daytime sleepiness and hypersomnia. In Sleep and Quality of Life in Clinical Medicine (eds JC Verster, SR Pandi-Perumal, DL Streiner): 107-18. Humana Press.

Montplaisir J, Boucher S, Nicolas A, et al (1998) Immobilization tests and periodic leg movements in sleep for the diagnosis of restless leg syndrome. Movement Disorders, 13: 324-9.

Moore TJ, Glenmullen J, Mattison DR (2014) Reports of pathological gambling, hypersexuality, and compulsive shopping associated with dopamine receptor agonist drugs. JAMA Internal Medicine, 174 1930-3.

Morgenthaler TI, Lee-Chiong T, Alessi C, et al (2007) Practice parameters for the clinical evaluation and treatment of circadian rhythm sleep disorders. An American Academy of Sleep Medicine report. Sleep, 30: 1445-59.

Myers E, Startup H, Freeman D (2011) Cognitive behavioural treatment of insomnia in individuals with persistent persecutory delusions: a pilot trial. Journal of Behavior Therapy and Experimental Psychiatry, 42: $330-6$.

Ohayon MM, Guilleminault C, Priest RG (1999) Night terrors, sleepwalking, and confusional arousals in the general population: their frequency and relationship to other sleep and mental disorders. Journal of Clinical Psychiatry, 60: 268-76.

Okuji Y, Matsuura M, Kawasaki N, et al (2002) Prevalence of insomnia in various psychiatric diagnostic categories. Psychiatry and Clinical Neurosciences, 56: 239-40.

Pollack M, Kinrys G, Krystal A, et al (2008) Eszopiclone coadministered with escitalopram in patients with insomnia and comorbid generalized anxiety disorder. Archives of General Psychiatry, 65: 551-62.

Postuma RB, Gagnon JF, Tuineaig M, et al (2013) Antidepressants and REM sleep behavior disorder: isolated side effect or neurodegenerative signal? Sleep, 36: 1579-85

Riemann D, Perlis ML (2009) The treatments of chronic insomnia: a review of benzodiazepine receptor agonists and psychological and behavioral therapies. Sleep Medicine Reviews, 13: 205-14.

Roth T (2007) Insomnia: definition, prevalence, etiology, and consequences. Journal of Clinical Sleep Medicine, 3(Suppl 5): 3-6.

Rottach KG, Schaner BM, Kirch MH, et al (2008) Restless legs syndrome as side effect of second generation antidepressants. Journal of Psychiatric Research, 42, 70-5.

Taylor DJ, Pruiksma KE (2014) Cognitive and behavioural therapy for insomnia (CBT-I) in psychiatric populations: a systematic review. International Review of Psychiatry, 26: 205-13.

Tek C, Palmese LB, Krystal AD, et al (2014) The impact of eszopiclone on sleep and cognition in patients with schizophrenia and insomnia: a double-blind, randomized, placebo-controlled trial. Schizophrenia Research, 160: $180-5$

Wirz-Justice A (2008) Diurnal variation of depressive symptoms. Dialogues in Clinical Neuroscience, 10: 337-43.

Wulff K, Gatti S, Wettstein JG, et al (2010) Sleep and circadian rhythm disruption in psychiatric and neurodegenerative disease. Nature Reviews Neuroscience, 11: 589-99. 
MCOs

Select the single best option for each question stem

1 In comorbid depression and insomnia:

a treating the insomnia is not necessary if the depression is adequately treated

b treating the insomnia will not have any impact on the depression

c CBT-I may lead to improvements in depressive symptoms

d the insomnia should be seen as a symptom of the depression

e SSRIs do not exacerbate insomnia.

2 In circadian rhythm sleep-wake disorders:

a the disordered sleep pattern is the result of lifestyle choices

b melatonin and light treatment can be effective interventions c going to bed at the same time every night is the most important intervention

$\mathrm{d}$ delayed sleep-wake phase disorder is more common in the elderly

e these disorders are rarely misdiagnosed as insomnia.

3 Of the following psychotropics, the one not known to precipitate or worsen sleep movement disorders is:

a mirtazapine

b olanzapine

c clozapine

d bupropion

e risperidone.

4 Of the following conditions, the one not classified as a non-REM parasomnia is:

a confusional arousa b sleepwalking

c sleep eating

d sleep terrors

e nightmare disorder.

5 Symptoms characteristic of hypersomnolence disorder include:

a hypnogogic hallucinations

b sleep inertia/drunkenness

c sleep paralysis

d hypnopompic hallucinations

e cataplexy. 